\title{
Lagrangian statistics and temporal intermittency in a shell model of turbulence
}

\author{
G. Boffetta, F. De Lillo, and S. Musacchio \\ Dipartimento di Fisica Generale and INFM, Università di Torino, Via Pietro Giuria 1, 10125 Torino, Italy \\ and CNR-ISAC-Sezione di Torino, Corso Fiume 4, 10133 Torino, Italy
}

(Received 16 July 2002; published 19 December 2002)

\begin{abstract}
We study the statistics of single-particle Lagrangian velocity in a shell model of turbulence. We show that the small-scale velocity fluctuations are intermittent, with scaling exponents connected to the Eulerian structure function scaling exponents. The observed reduced scaling range is interpreted as a manifestation of the intermediate dissipative range, as it disappears in a Gaussian model of turbulence.
\end{abstract}

DOI: 10.1103/PhysRevE.66.066307

PACS number(s): 47.27.Gs, 47.27.Qb

In recent years there has been a great improvement in the laboratory experimental investigation of turbulence from a Lagrangian point of view [1-4]. In the Lagrangian approach, the flow is described by the (Lagrangian) velocity $\boldsymbol{v}\left(\boldsymbol{x}_{0}, t\right)$ of a fluid particle initially at position $\boldsymbol{x}(0)=\boldsymbol{x}_{0}$. This is the natural description for studying transport and mixing of neutrally advected substances in turbulent flows.

One of the simplest statistical quantities one can be interested in is single-particle velocity increments $\delta \boldsymbol{v}(t)=\boldsymbol{v}(t)$ $-\boldsymbol{v}(0)$ (where, assuming statistical homogeneity, we have dropped the dependence on $\boldsymbol{x}_{0}$ ) for which dimensional analysis in fully developed turbulence predicts $[5,6]$

$$
\left\langle\delta v_{i}(t) \delta v_{j}(t)\right\rangle=C_{0} \varepsilon t \delta_{i j},
$$

where $\varepsilon$ is the mean energy dissipation and $C_{0}$ is a numerical constant. The remarkable coincidence that the variance of $\delta \boldsymbol{v}(t)$ grows linearly with time is the physical basis on which stochastic models of particle dispersion are based. It is important to recall that the "diffusive" nature of Eq. (1) is purely incidental: it is a direct consequence of Kolmogorov scaling in the inertial range of turbulence and is not directly related to a diffusive process. Let us recall briefly the argument leading to the scaling in Eq. (1). We can think of the velocity $v(t)$ advecting the Lagrangian trajectory as the superposition of the different velocity contributions coming from turbulent eddies (which also move with the same velocity of the Lagrangian trajectory). After a time $t$ the components associated with the smaller (and faster) eddies, below a certain scale $\ell$, are decorrelated and thus at the leading order one has $\delta v(t) \simeq \delta v(\ell)$. Within Kolmogorov scaling, the velocity fluctuation at scale $\ell$ is given by $\delta v(\ell)$ $\sim V(\ell / L)^{1 / 3}$, where $V$ represents the typical velocity at the largest scale $L$. The correlation time of $\delta v(\ell)$ scales as $\tau(\ell) \sim \tau_{0}(\ell / L)^{2 / 3}$ and thus one obtains the scaling in Eq. (1) with $\varepsilon=V^{2} / \tau_{0}$.

This argument shows that the linear scaling in Eq. (1) is the result of the combination of the Kolmogorov scaling for velocity fluctuations and eddy turnover time in physical space, as seen by a Lagrangian tracer. From a numerical point of view, the observation of Eq. (1) is more delicate than standard Eulerian structure functions, as it requires the correct resolution of the sweeping effect on the Lagrangian trajectories. Of course, this can be done in direct numerical simulations (but at moderate Reynolds numbers) [7] and, as we will see, in a Lagrangian version of the shell model of turbulence.

Equation (1) can be generalized to higher-order moments with the introduction of a set of temporal scaling exponents $\xi(p)$,

$$
\left\langle\delta v(t)^{p}\right\rangle \sim V^{p}\left(t / \tau_{0}\right)^{\xi(p)} .
$$

The dimensional estimation sketched above gives the prediction $\xi(p)=p / 2$ but one might expect corrections to dimensional scaling in the presence of intermittency.

A generalization of Eq. (1) which takes into account intermittency corrections can be easily developed by extending the previous argument within the multifractal model of turbulence $[8,9]$. The dimensional argument is repeated for the local scaling exponent $h$, giving $\delta v(t) \sim V\left(t / \tau_{0}\right)^{h /(1-h)}$. Integrating over the $h$ distribution one ends with

$$
\left\langle\delta v(t)^{p}\right\rangle \sim V^{p} \int d h\left(\frac{t}{\tau_{0}}\right)^{[p h-D(h)+3] /(1-h)} .
$$

In the limit $t / \tau_{0} \rightarrow 0$, the integral can be estimated by a steepest descent argument giving the prediction

$$
\xi(p)=\min _{h}\left[\frac{p h-D(h)+3}{1-h}\right] \text {. }
$$

The fractal dimension $D(h)$ is related to the Eulerian structure function scaling exponents $\zeta(q)$ by the Legendre transform [9] $\zeta(q)=\min _{h}[q h-D(h)+3]$. The standard inequality in the multifractal model [following from the exact result $\zeta(3)=1], D(h) \leqslant 3 h+2$, implies for Eq. (4) that even in presence of intermittency $\xi(2)=1$. This is a direct consequence of the fact that energy dissipation enters into Eq. (1) at the first power. Our expression for scaling exponents (4) recovers in a more compact form the prediction obtained on the basis of an "ergodic hypothesis" of the statistics of energy dissipation [10].

Recent experimental results [4] have shown that indeed Lagrangian velocity fluctuations are intermittent and display anomalous scaling exponents, as predicted by the above argument. Despite the relative high Reynolds number of the experiments, a true temporal scaling range is not observed. 
Thus the estimation of the scaling exponents in Eq. (2) can be done only relatively to a reference moment (the so-called ESS procedure [11]).

In this work we use a dynamical shell model of turbulence for investigating scaling (2) and prediction (4) at very high Reynolds numbers. In shell models the velocity fluctuations of the eddies at the scale $\ell_{n}=L 2^{-n}$ are represented by a single variable $u_{n}(n=1,2, \ldots, N)$. Only local interactions among shells are represented and therefore no sweeping effects are present [12]. In this sense shell models are dynamical models of velocity fluctuations in a Lagrangian framework, and for this reason have been already used in the study of turbulent dispersion [13]. The equation of motion for the complex shell variable $u_{n}$ is [14]

$$
\begin{aligned}
\frac{d u_{n}}{d t}= & i k_{n}\left(u_{n+2} u_{n+1}^{*}-\frac{\delta}{2} u_{n+1} u_{n-1}^{*}+\frac{1-\delta}{4} u_{n-1} u_{n-2}\right) \\
& -\nu k_{n}^{2} u_{n}+f_{n},
\end{aligned}
$$

where $k_{n}=\ell_{n}^{-1}, f_{n}$ is a deterministic forcing acting on the first shells only, $\nu$ is the viscosity, and $\delta$ is a parameter. Shell model (5) is characterized by a chaotic dynamics with a statistically steady state with a constant flux of kinetic energy from large scales to small scales. The fluctuations generated by the chaotic dynamics are responsible for the observed corrections to the Kolmogorov exponents for the structure functions which are found close to the accepted experimental values [12].

Lagrangian velocity in the shell model framework can be represented as the superposition of the contributions of all the different eddies. Let us define

$$
v(t) \equiv \sum_{n=1}^{N} \operatorname{Re}\left(u_{n}\right)
$$

where we have taken, rather arbitrarily, only the real part of the shell variables with a unit coefficient. Indeed, from the definition of the shell model, there is no precise recipe for reconstructing the Lagrangian velocity. More generally, one could think of a representation in which shell variables are multiplied by appropriate wavelet functions. Of course, numerical prefactors such as $C_{0}$ in Eq. (1) will depend on the wavelet basis and thus cannot be estimated within the present approach. Nevertheless one expects that different choices for the basis do not affect Lagrangian scaling exponents $\xi(p)$, which are determined by the dynamical properties of the model.

Previous studies of multitime correlations in shell models of turbulence have shown the existence of a set of correlation times, in agreement with the multifractal picture of the turbulent cascade [15]. This is an indication that, as we will see, the Lagrangian velocity defined as Eq. (6) will be affected by intermittency.

Very long and accurate numerical simulations of the shell model (5) with $N=24$ shells and $\delta=1 / 2$ have been performed. The energy is injected at a constant flux $\varepsilon=0.01$ in the first two shells and is removed at the smallest shells by viscosity $\nu=10^{-7}$. With these parameters, our simulations

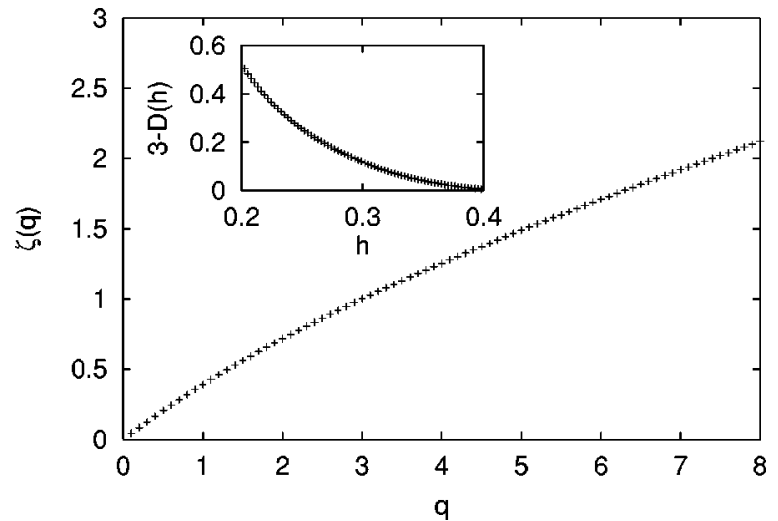

FIG. 1. Shell model Eulerian structure function scaling exponents $\zeta(q)$ determined from the statistics over $10^{5}$ independent configurations. In the inset we plot the codimension $3-D(h)$ as determined by numerically inverting the Legendre transform.

correspond to a Reynolds number $\operatorname{Re} \simeq 10^{8}$. For each realization, Lagrangian structure functions are computed from the Lagrangian velocity (6) up to the large-scale time $\tau_{0}$. An average is then taken over $10^{5}$ independent realizations. The Eulerian scaling exponents $\zeta(q)$ are determined within the same simulations from the Eulerian structure functions $S_{q}\left(k_{n}\right)=\left\langle\left|u_{n}\right|^{q}\right\rangle \sim k_{n}^{-\zeta(q)}$.

In Fig. 1 we plot the set of numerically determined Eulerian structure function scaling exponents $\zeta(q)$ together with the fractal dimension $D(h)$ reconstructed by inverting the Legendre transform. We observe strong intermittency in velocity statistics with scaling exponents that clearly deviate from the Kolmogorov prediction. We remark that the scaling exponents are not universal with respect to the particular shell model. Model (5) gives a set of exponents which are a little more intermittent than, but not far from, the experimentally observed exponents [9]: $\zeta(2) \simeq 0.72, \quad \zeta(4) \simeq 1.25$, $\zeta(6) \simeq 1.71$. As a consequence, the values of $\xi(p)$ obtained from Eq. (4) using the $D(h)$ of Fig. 1 can be compared directly with experimental data results.

Figure 2 shows the second-order Lagrangian structure function (1) as a function of time. The linear behavior is evident (see the inset) even if a long crossover from the ballistic scaling at short time $\left\langle\delta v(t)^{2}\right\rangle \sim t^{2}$ is present. In spite of the very high Reynolds numbers achievable in the shell model, the extension of the temporal scaling (2) is still moderate. For a comparison with the available experimental data, in the inset we also plot the result obtained from two simulations at lower resolution, with $\operatorname{Re} \simeq 2 \times 10^{6}$ and $\operatorname{Re} \simeq 10^{5}$. In the latter case almost no scaling range is observable. Despite these limitations, we will see that high Re simulations allow the determination of the Lagrangian scaling exponents with good accuracy.

In Fig. 3 we plot the probability density functions (pdf's) of $\delta v(t)$ computed at different $t$ in the linear scaling range of Fig. 2 rescaled with their variances. The form of the pdf varies continuously from almost Gaussian at large time $\left(t \sim \tau_{0}\right)$ to the development of stretched exponential tails at short times, similar to what was observed in laboratory experiments [4]. Flatness $F$ grows from the Gaussian value 


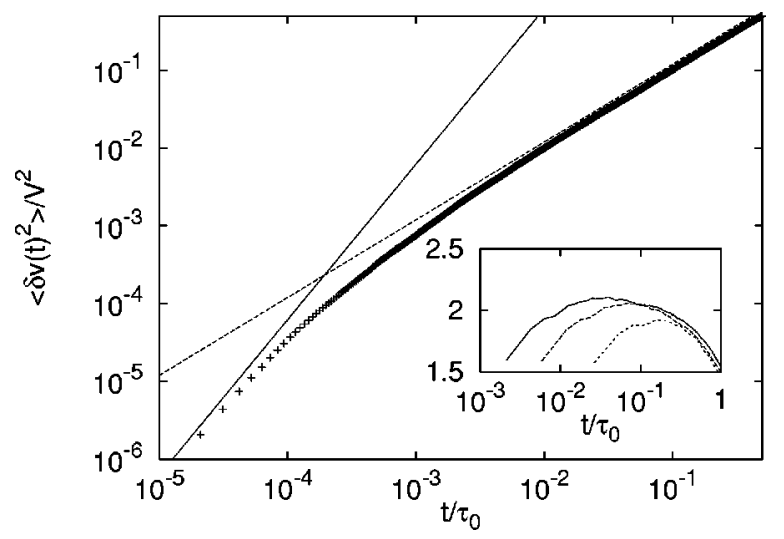

FIG. 2. Second-order Lagrangian structure function $\left\langle\delta v(t)^{2}\right\rangle$ normalized with large-scale velocity $V$ as a function of time delay $t$ for the simulation at $\operatorname{Re}=10^{8}$. The continuous line is the ballistic behavior $t^{2}$ at short time. The dashed line represents the linear growth (1). Inset: $\left\langle\delta v(t)^{2}\right\rangle$ compensated with the dimensional prediction $\varepsilon t$ at $\mathrm{Re}=10^{8}$ (continuous line), $\mathrm{Re}=2 \times 10^{6}$ (dashed line), and $\operatorname{Re}=10^{5}$ (dotted line).

$F=3$ up to $F \simeq 20$ at smallest times. This is an indication of Lagrangian intermittency, in the sense that the Lagrangian statistics cannot be described in terms of a single scaling exponent.

In Fig. 4 we plot the set of Lagrangian scaling exponents $\xi(p)$ obtained from a direct fit of temporal structure functions. The nonlinear behavior in $p$ confirms the presence of Lagrangian intermittency already observed from the pdf. We present the result for moments up to $p=6$, which approximately corresponds, from Eq. (4), to the Eulerian structure function of order $q=8$. In this sense temporal structure functions are more intermittent. Figure 4 shows that the agreement with the multifractal prediction (4) is very good up to the moment achievable with our statistics. What is even more remarkable is that our prediction is very close to experimentally determined exponents. For example, we find $\xi(3)$

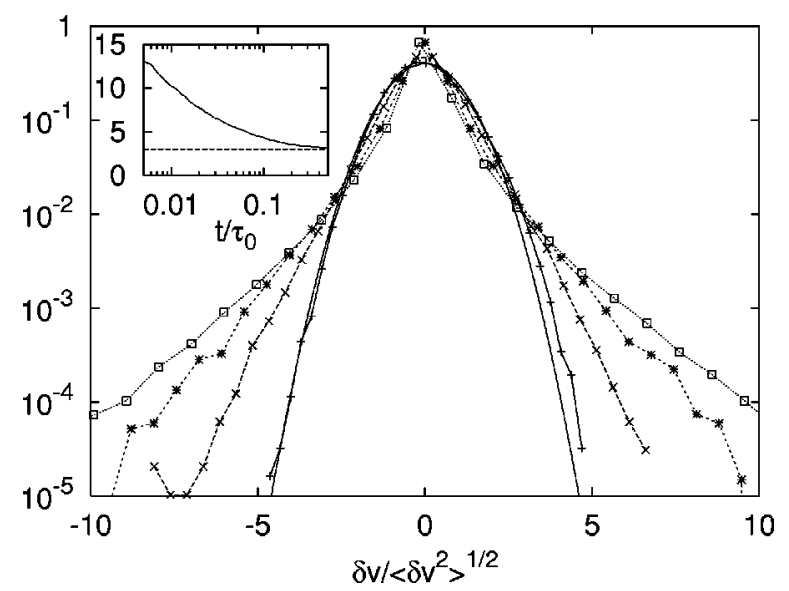

FIG. 3. Probability density functions of velocity differences $\delta v(t)$ normalized with the variance at time lags $t / \tau_{0}$ $=0.002(\square), 0.01(*), 0.06(\times), 0.35(+)$. The continuous line represents a Gaussian. Inset: flatness $F=\left\langle\delta v(t)^{4}\right\rangle /\left\langle\delta v(t)^{2}\right\rangle^{2}$ as a function of time and Gaussian value $F=3$ (dashed line).

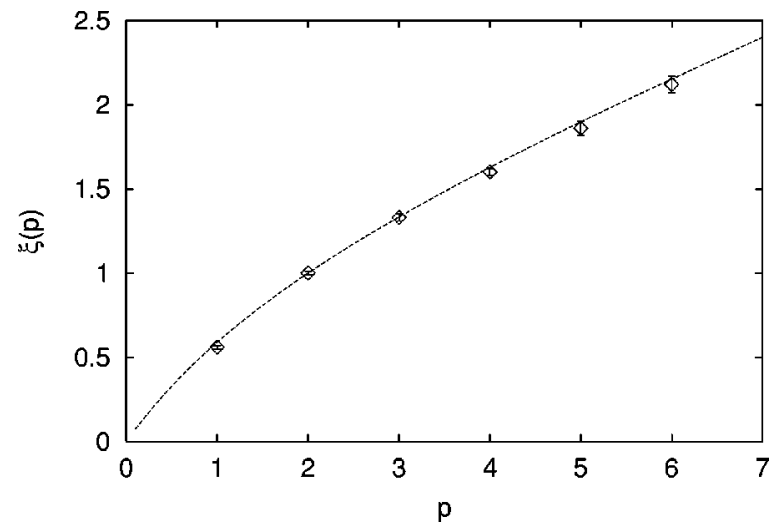

FIG. 4. Lagrangian structure function scaling exponents $\xi(p)$ numerically determined by a best fit on Eq. (2). The line represents the multifractal prediction (4) with $D(h)$ obtained from Fig. 1.

$\simeq 1.31, \xi(4) \simeq 1.58, \xi(5) \simeq 1.85$, while the experimental data give [4] $\xi_{\text {exp }}(3)=1.34 \pm 0.02, \quad \xi_{\text {exp }}(4)=1.56 \pm 0.06$, and $\xi_{\text {exp }}(5)=1.8 \pm 0.2$. Of course our prediction (4) should be directly tested by using the fractal dimension $D(h)$ obtained from the experimental data.

We conclude with a brief discussion on the effects of intermittency on the extension of the scaling range. The long crossover in Fig. 2 can be understood in terms of the intermediate dissipative range as a consequence of the fluctuating dissipative scale $[9,16]$. Indeed, the smallest time at which one can expect scaling (1) is the Kolmogorov time $\tau_{\eta}$ $\sim \tau_{0} \operatorname{Re}^{-(1-h) /(1+h)}$, which fluctuates with $h$. A demonstration of the effects induced by intermittency is given by considering a nonintermittent Gaussian model.

Setting $f_{n}=\nu=0$, Eq. (5) becomes a conservative system with two conserved quantities which depend on the value of $\delta$ [12]. In statistically stationary conditions, the model shows equipartition of the conserved quantities among the shells, in agreement with the statistical mechanics prediction [17]. For $\delta=1+2^{-2 / 3}$ the equipartition state leads at small scales to Kolmogorov scaling $\left\langle\left|u_{n}\right|^{2}\right\rangle \sim k_{n}^{-2 / 3}$ with Gaussian statistics.

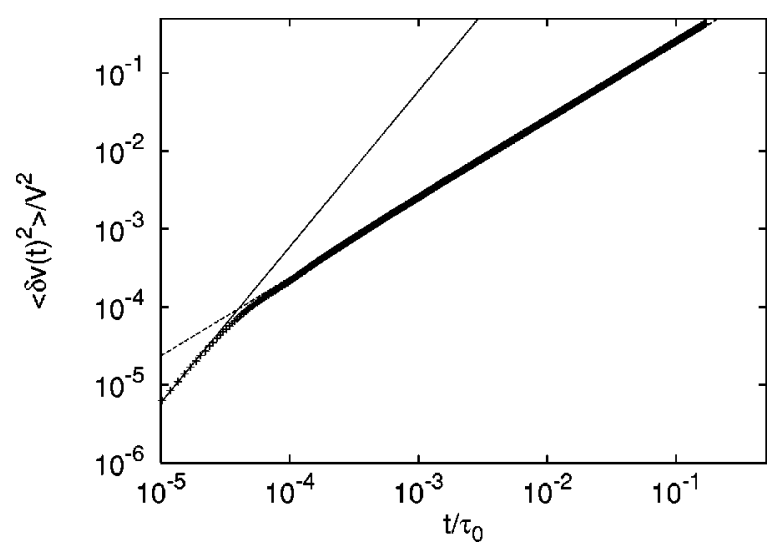

FIG. 5. Second-order Lagrangian structure function $\left\langle\delta v(t)^{2}\right\rangle$ normalized with large-scale velocity $V$ as a function of time delay $t$ for the equilibrium Gaussian model. The continuous line is the ballistic behavior $t^{2}$ at short time. The dashed line represents the linear growth (1). 
In Fig. 5 we plot the second-order Lagrangian structure function (1) for the Gaussian model. Both ballistic and diffusive scaling are clearly observable and the crossover is strongly reduced with respect to Fig. 2 .

In this work we have investigated the statistical properties of Lagrangian velocity in fully developed turbulence. A prediction for intermittent scaling exponents of Lagrangian structure functions is given within the multifractal formalism. Very high Reynolds number simulations in the shell model confirm the multifractal prediction, even if rather small scaling ranges are observed. At lower Reynolds numbers, comparable with those achievable in laboratory experi- ments, almost no scaling is observed and the scaling exponents can be determined only in a relative way. The reduction of the scaling range in Lagrangian statistics is interpreted as an effect of the intermediate dissipative range. A Gaussian, nonintermittent version of the shell model confirms this interpretation. Deviations from Gaussianity in Lagrangian statistics impose severe limitations on the use of stochastic models for particle dispersion [18], which should be modified in order to take into account the effects of intermittency.

This work was supported by MIUR-Cofin2001 Contract No. 2001023848. We acknowledge the allocation of computer resources from INFM "Progetto Calcolo Parallelo."
[1] G.A. Voth, K. Satyanarayan, and E. Bodenschatz, Phys. Fluids 10, 2268 (1998).

[2] S. Ott and J. Mann, J. Fluid Mech. 422, 207 (2000).

[3] A. La Porta, G.A. Voth, A.M. Crawford, J. Alexander, and E. Bodenschatz, Nature (London) 409, 1017 (2001).

[4] N. Mordant, P. Metz, O. Michel, and J.F. Pinton, Phys. Rev. Lett. 87, 214501 (2001).

[5] A.S. Monin and A.M. Yaglom, Statistical Fluid Mechanics (MIT Press, Cambridge, MA, 1975).

[6] H. Tennekes and J.L. Lumley, A First Course in Turbulence (MIT Press, Cambridge, MA, 1972).

[7] P.K. Yeung, J. Fluid Mech. 427, 241 (2001).

[8] G. Parisi and U. Frisch, in Turbulence and Predictability in Geophysical Fluid Dynamics, Proceedings of the International School of Physics "Enrico Fermi," 1983, edited by M. Ghil, R. Benzi, and G. Parisi (North-Holland, Amsterdam, 1985).

[9] U. Frisch, Turbulence (Cambridge University Press, Cambridge, U.K., 1995).
[10] M.S. Borgas, Proc. R. Soc. London, Ser. A 342, 379 (1993).

[11] R. Benzi, S. Ciliberto, R. Tripiccione, C. Baudet, F. Massaioli, and S. Succi, Phys. Rev. E 48, R29 (1993).

[12] T. Bohr, M. Jensen, G. Paladin, and A. Vulpiani, Dynamical Systems Approach to Turbulence (Cambridge University Press, Cambridge, U.K., 1998).

[13] G. Boffetta, A. Celani, A. Crisanti, and A. Vulpiani, Phys. Rev. E 60, 6734 (1999).

[14] V.S. L'vov, E. Podivilov, A. Pomyalov, I. Procaccia, and D. Vandembroucq, Phys. Rev. E 58, 1811 (1998).

[15] L. Biferale, G. Boffetta, A. Celani, and F. Toschi, Physica D 127, 187 (1999).

[16] U. Frisch and M. Vergassola, Europhys. Lett. 14, 439 (1991).

[17] E. Aurell, G. Boffetta, A. Crisanti, P. Frick, G. Paladin, and A. Vulpiani, Phys. Rev. E 50, 4705 (1994).

[18] D.J. Thomson, J. Fluid Mech. 180, 529 (1987). 